\title{
EXPERIENCE WITH A STATIC MAGNETOSTRICTIVE READOUT SYSTEM FOR WIRE CHAMBERS*
}

\author{
M. J. LONGO, J. CLARK, G. EDICT, M. FOLLEBOUT ${ }^{\dagger}$ and H. R. GUSTAFSON \\ University of Michigan, Ann Arbor, Michigan 48104, U.S.A.
}

R. W. KELLER, L. M. SOMPAYRAC and K. K. YOUNG

University of Washington, Seattle, Washington 98105, U.S.A.

Received 1 March 1971

\begin{abstract}
Bespalova et al. have described a scheme for wire chamber readout which makes use of the permanent magnetization of the magnetostrictive line for temporary storage of coordinate information. The advantage of this scheme is that it can significantly reduce the cost of the electronics needed to read out a system with many chambers. We have adopted this technique to read out
\end{abstract}

\section{Introduction}

Bespalova et al. ${ }^{1}$ ) have described a scheme for wire chamber readout which makes use of the permanent magnetization of the magnetostrictive (MS) line for temporary storage of the coordinate information. Monds and Rosie $^{2}$ ) have discussed the application of this technique for storage of information but it has not been widely applied to spark chambers. This delayed readout system has been called a "static" readout system to distinguish it from the more common prompt or "dynamic" readout in which the acoustic pulses set up immediately on the line by the current in the spark chamber wires are read out.

This technique can be best understood by referring to fig. 1, which schematically illustrates a magnetostrictive line and pickup coil. In the more common dynamic readout system the magnetic field from a current pulse in one of the chamber wires produces a local magnetostrictive strain in the MS line. This disturbance propagates down the line at the velocity of sound and is detected by the pickup coil. Usually fiducial wires are pulsed at the same time as the chamber wires so several pulses are set up in the line simultaneously. The time interval between the leading fiducial and subsequent pulses is measured. Usually a system of scalers driven from a master oscillator is used with the scalers all started by the first pulse and then each scaler in turn stopped by subsequent pulses. Thus to provide the capability of reading out four spark locations requires a bank of four or more scalers per line. With an experiment requiring many

\footnotetext{
* This work was sponsored by the U.S. National Science Foundation and the Atomic Energy Commission.

$\dagger$ Deceased.
}

spark coordinates in an array of spark chambers to detect $\gamma$ showers. This array has been used successfully in an experiment in which $\approx 10^{7}$ events were recorded. We discuss our experience with this type of readout and its advantages and disadvantages relative to the more common "prompt" readout system.

lines a very large number of scalers and associated discriminators are needed.

In the static readout mode the arrangement is the same except for the addition of the current tap shown in fig. 1. It is assumed that the line is originally uniformly magnetized along its length by sending a current pulse down the line. Pulsing the chamber wires sets up the usual dynamic pulse which is disregarded. It also sets up a "patch" of permanent magnetization on the line. The position of this patch can later be read at any desired time by sending a current pulse down the line from the current tap. The interaction between the magnetic field set up by the readout current and that of the patch generates a strain pulse in the line which can be read out in the same way as in the dynamic readout.

The main advantage of the static readout is that a number of lines can be interrogated sequentially with their outputs going to the same bank of scalers. (The outputs of the preamplifiers are summed with a simple "or" circuit.) We have found that the interrogating pulse can be supplied by an inexpensive SCR circuit.

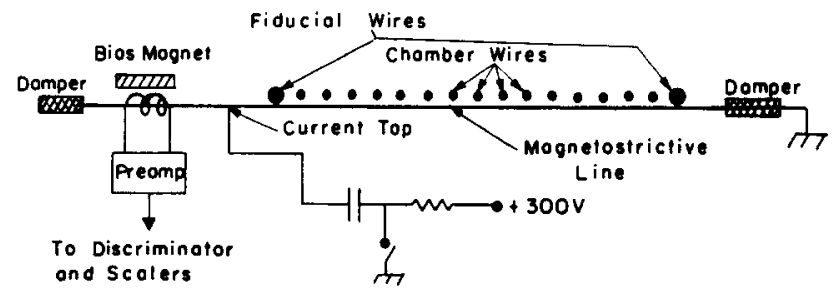

Fig. 1. Schematic of static readout system. Patches of permanent magnetization are left on the line by the current in the chamber and fiducial wires. The readout is initiated by sending a current pulse down the MS line from the current tap. 
TABLE 1

Dynamic readout Static readout

$\begin{array}{lcc}\text { Signal size } & \approx 20 \mathrm{mV} & \approx 2 \mathrm{mV} \\ \text { Noise } & \text { quite small } & \approx 0.2 \mathrm{mV} \\ \begin{array}{l}\text { Sensitivity to } \\ \text { external } \\ \text { magnetic field }\end{array} & \text { OK to } \approx 100 \mathrm{~g} & \text { OK to } \approx 20 \mathrm{~g} \\ \begin{array}{l}\text { Spatial accuracy } \\ \text { Readout time }\end{array} & \begin{array}{c}\text { typically } \approx 100 \\ \mu \text { sec independent } \\ \text { of no. of chambers }\end{array} & \begin{array}{c}\text { (no. of chambers) } \\ \times 100 \mu \mathrm{sec}\end{array} \\ & \end{array}$

If the interrogating current is large enough, the information is erased completely and the line is uniformly magnetized, ready for a new event. A noteworthy feature of the static readout system is that it can make use of many of the techniques and electronic circuits developed for the dynamic readout with little modification.

We have developed a static readout system for a gamma shower detector for an experiment to measure differential cross sections for the reaction $n+p \rightarrow \gamma+d$. The gamma detector consists of an array of 28 singlegap wire chamber modules interspersed with lead plates and triggering counters. The function of this array is to convert $\gamma$ 's with high efficiency and to measure the position of the vertex of the shower. The required spatial resolution in the vertex position was $\approx \pm 2 \mathrm{~mm}$. The active area of each chamber was $48 \times 84 \mathrm{~cm}$. Each wire chamber has a set of vertical and horizontal wires. Though "spark" counting to determine the $\gamma$ energy was not planned, it was felt that there should be the capability of reading out at least four sparks per line to simplify vertex recognition. To do this with a dynamic readout would require a system with 224 scalers, which would have been prohibitively expensive. We therefore chose to employ a static readout system in which the outputs from the $56 \mathrm{MS}$ lines would be summed into two groups of 28 each. Each group would then go to a separate bank of 6 scalers (giving us in principle a 6 spark capability). Each of the lines could then be interrogated sequentially under computer control.

\section{Comparison of the static and dynamic systems}

The main advantage of the static system is the possibility of a considerable saving in electronics especially when a large number of lines and several sparks per line are to be read out. There are, however, significant disadvantages which must be considered for each application. These are summarized in table 1 and will be briefly discussed here.

\subsection{Signal SIZE}

The static pulses are typically about an order of magnitude smaller than the dynamic pulses. This necessitates the use of an MS line of larger cross section, higher gain preamps, and special heat treatment of the ribbons. The most appropriate combination of these depends on the circumstances. We shall discuss our choice below.

\subsection{NOISE}

Noise pulses can be generated in the lines by the magnetic field produced by the interrogating current. These pulses can be set up anywhere along the ribbon at discontinuities of any sort, such as kinks, nicks, or changes in permeability. Careful handling of the ribbons minimizes such noise pulses. Our best signalto-noise ratios have been $\approx 20: 1$, but this depends critically on heat treatment, current in the chamber wires, ribbon size, etc. A large noise pulse is also generated at the current tap (fig. 1), but this can easily be gated out electronically and is not a problem.

\subsection{Sensitivity to EXternal MaGnetic FieldS}

The static readout is more sensitive to external magnetic fields. The sensitivity depends on annealing but typically a longitudinal field of $15 \mathrm{G}$ will halve the pulse height (fig. 2). The noise from the line also increases with the external field, causing a rapid detcrioration of the signal-to-noise ratio. Furthermore the static readout is very sensitive to local gradients in the field since these cause dynamic pulses to be set up when the lines are interrogated. This makes magnetic

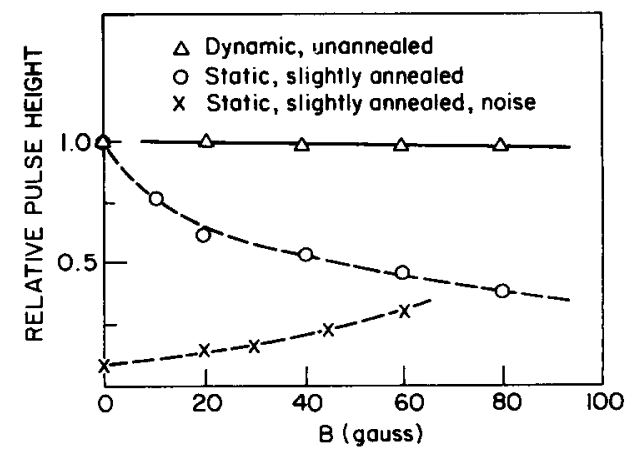

Fig. 2. Typical variation of output pulse height and noise with longitudinal magnetic field. The ribbon used for the dynamic readout was hard drawn; that used for the static readout was partially annealed. 
shielding more difficult. In the $\mathrm{n}+\mathrm{p} \rightarrow \gamma+\mathrm{d}$ experiment the chambers operated in the stray magnetic field of the LRL 184" cyclotron which in some chamber positions was as large as $60 \mathrm{G}$. This necessitated shielding the whole array in an iron box.

\subsection{Spatial accuracy}

Bespalova et al. ${ }^{1}$ ) reported that the static system is superior to the dynamic system in spatial accuracy. However our experience indicates the reverse is likely to be true. We do not yet have direct measurements of the resolution possible with a static readout. With the dynamic readout using $0.15 \mathrm{~mm}$ diameter unannealed ribbon we have consistently obtained pulse widths $<0.5 \mu \mathrm{sec}$. The static pulses are also more variable in shape, so it seems likely that the resolution will be 2 to 3 times poorer.

\subsection{ReAdout TIME}

Because the ribbons are interrogated sequentially the time required to read out the system is increased. The total readout time is essentially the sum of that required to read out each of the ribbons in the sequence.

\section{Details of the system used in the $\mathbf{n}+\mathbf{p} \rightarrow \boldsymbol{y}+\mathbf{d}$ experiment}

\subsection{General Description}

The gamma detector consisted of 28 single-gap modules ( 56 wire planes) interspersed with lead plates and triggering counters. Each module has a set of vertical wires on one side and horizontal wires on the other with a spacing of $1 \mathrm{~mm}$ between wires. The dimensions of the active area of each chamber were $48 \times 84 \mathrm{~cm}$ with a $1.25 \mathrm{~cm}$ gap between the wire planes. The frames were made of G-10 epoxy fiberglas. Each module had mylar windows and could be tested independently of the others.

Each module was powered by a separate $6800 \mathrm{pF}$ capacitor charged to about $12.5 \mathrm{kV}$. The voltage was applied at the center of the bus connecting the wires in each plane. The buses were "terminated" at either end by $100 \Omega$ resistors connected between the H.V. bus and the ground bus. The current through the $100 \Omega$ resistors was also routed through fiducial wires near the end of each plane. It was found that spurious sparks in the absence of tracks could be reduced and chamber operation considerably improved by covering the outside of the mylar windows with aluminum foil which was electrically connected to the adjacent wire planes. We believe that without these foils large electric fields are produced between the wires and the mylar when the high voltage pulse is applied. This produces ion pairs which tend to cause spurious breakdowns.

The chambers were operated with the usual gas mixture of approximately $90 \%$ neon and the remainder helium. About $5 \%$ of the gas was passed over ethyl alcohol at room temperature. Under these operating conditions spurious sparks in the absence of a good track were quite rare, and reasonably good multispark capability was achieved.

\subsection{The MAGNETOSTRICTIVE LINES: HEAT TREATMENT}

The MS lines used were $0.10 \times 0.38 \mathrm{~mm}$ remendur (49\% iron, $49 \%$ cobalt, $2 \%$ vanadium) ribbons mounted against the chamber wires. Some studies of other possible magnetostrictive materials were made with little success. Remendur ribbons of smaller cross section were also tried, but these gave lower signal levels and considerably poorer signal-to-noise ratios. The remendur ribbon was partially annealed when purchased, but it was found that further heat treatment could significantly increase the pulse height. This was done by mounting the ribbon in an evacuated tube and passing a current of approximately $0.5 \mathrm{~A}$ through it for a few minutes. Monitoring the current through the ribbon was not sufficiently accurate to give reproducible results, so we used an infrared-sensitive photocell mounted close to the center of the ribbon to monitor the temperature more directly. The photocell reading which gave ribbons with optimum performance in the static readout mode was found by trial and error. Generally speaking the output of the ribbons increased as the current was raised up to a point, corresponding to a barely visible red glow, beyond which it decreased abruptly. However it was also found that the higher the annealing temperature the greater the attenuation of the MS pulses down the ribbon. The optimum heat treatment was therefore a compromise between increased pulse height and tolerable attenuation ${ }^{3}$ ). Typically after treatment the static pulse height was scveral times larger and the attenuation was $\approx 30 \%$ over a meter. The annealing also increased the dynamic pulses from the ribbons dramatically. Despite great care in handling, some of the ribbons had large noise pulses when interrogated and were rejected. The yield of good ribbons was $\approx 70 \%$.

\subsection{MOUNTING OF THE RIBBONS}

The ribbons were threaded through flattened $26 \mathrm{ga}$. tefion tubing and mounted in a groove on a long aluminum strip. A box containing the preamp, pickup coil, and bias magnet was rigidly attached to the strip 
to form an assembly which could easily be removed from the chambers for testing or replacement. This assembly is patterned after a Lawrence Radiation Laboratory design ${ }^{4}$ ). The preamp end of such a "wand" is shown partially dismantled in fig. 3 . The current tap was made by lightly soldering a piece of no. 34 wire to the ribbon just ahead of the pickup coil. The ground return was made by simply grounding the far end of the ribbon to the aluminum strip; the other end of the ribbon is insulated from ground. It is possible to put the current tap at the end of the ribbon so that the interrogating current flows through the pickup coil.

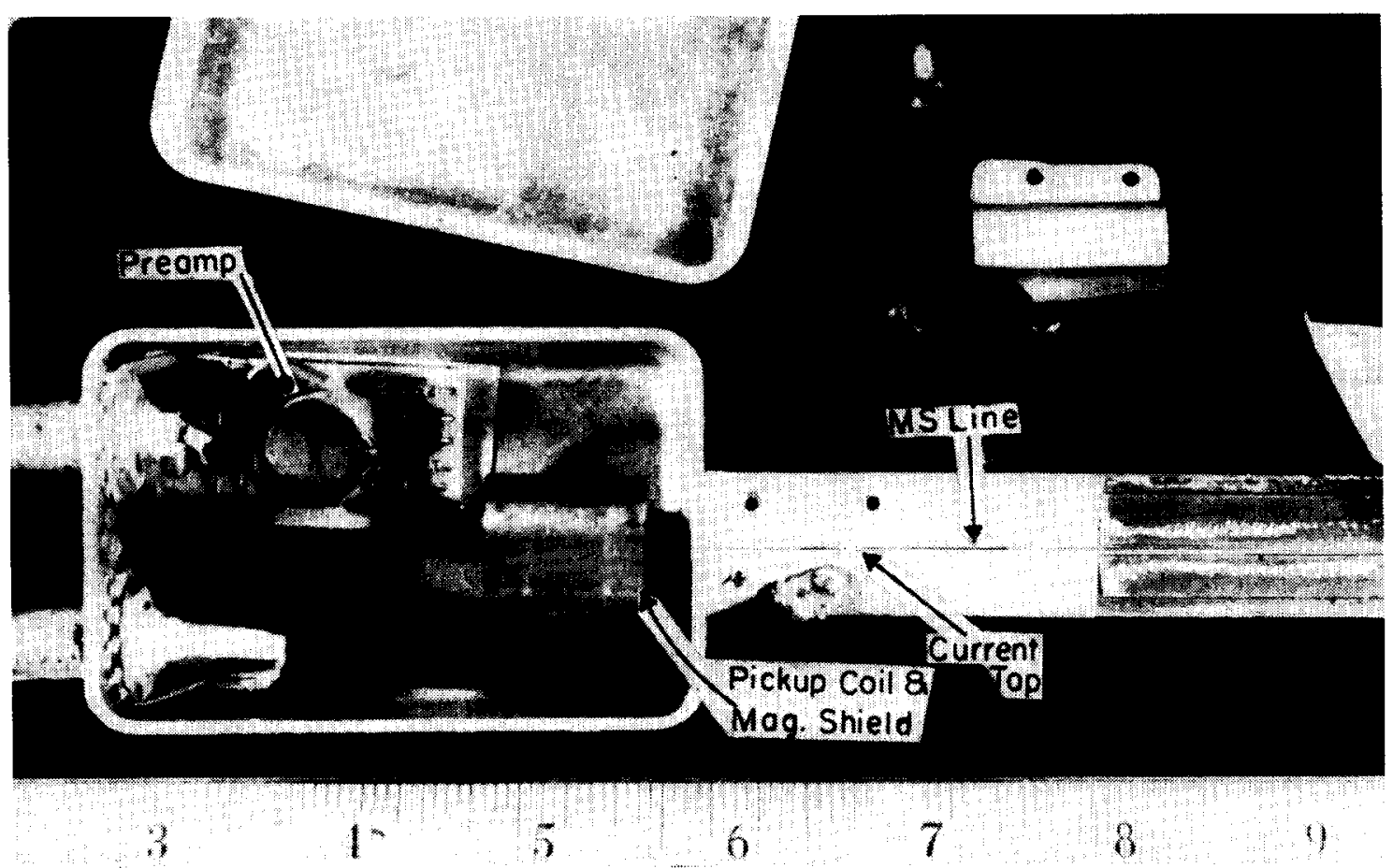

Fig. 3. The preamp end of a "wand" assembly showing the mounting of the ribbon, damping tube, current tap, preamp, etc.

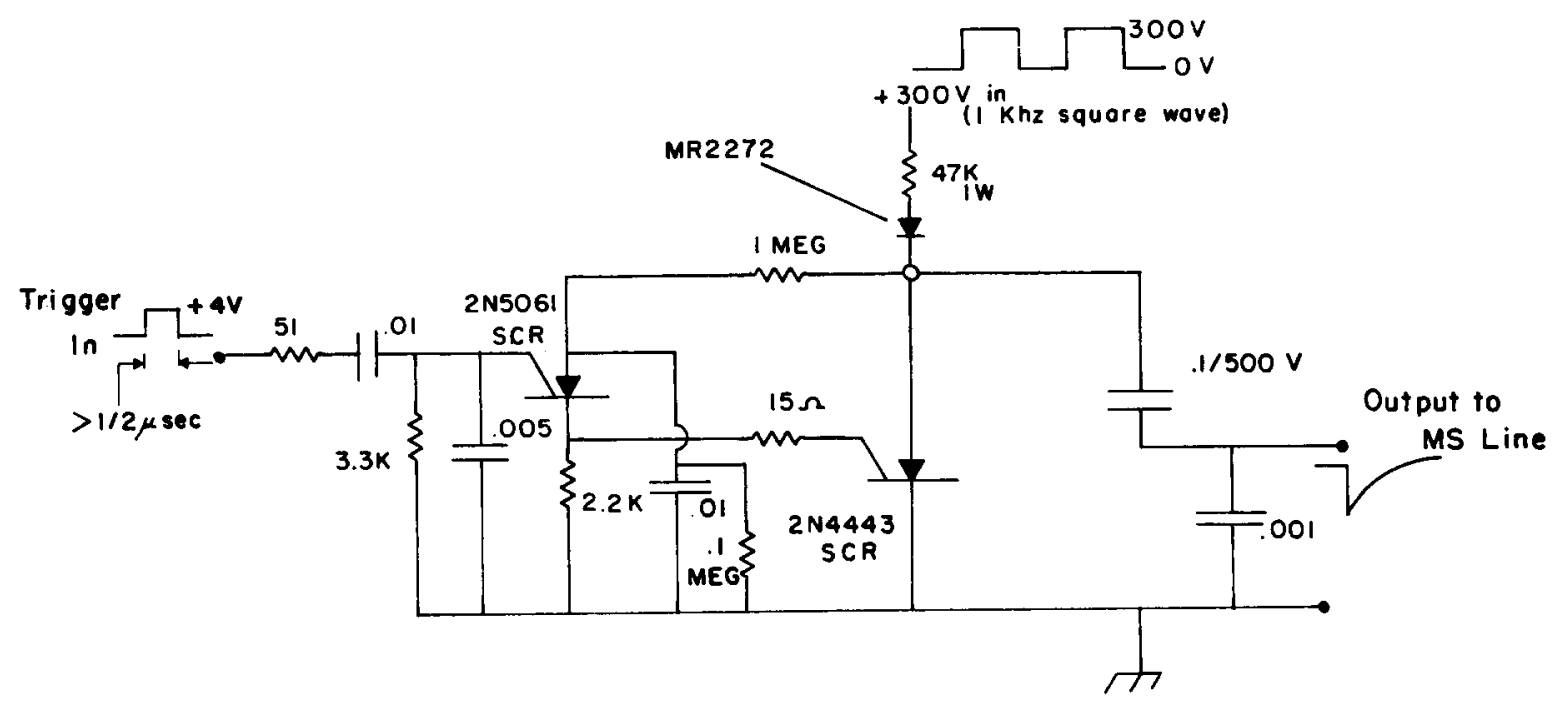

Fig. 4. The SCR pulser used for interrogating the wands. 
However with this system noise tends to be more of a problem and the large pulse induced in the pickup coil by the interrogating current causes severe overloading of the preamp. In fig. 3 the interrogating pulse is fed in one BNC connector; the other is the preamp output which also supplies $24 \mathrm{~V}$ dc to the preamp.

Damping of the pickup end of the ribbon had to be done very carefully to reduce reflections of the very large pulse produced at the current tap. This was done by passing the ribbon through a tube filled with vacuum grease on the coil side and Apiezon Q sealing compound on the side way from the coil. The far end was also damped by a similar arrangement except that a small quantity of vacuum grease and Apiezon $Q$ were put directly in the groove holding the ribbon. The amplitude of the pulses is fairly sensitive to tension on the ribbon, which was adjusted for maximum amplitude.

The pickup coil and bias magnet were similar to the L.R.L. design ${ }^{4}$ ). The preamp was also like that described in ref. 4 , except that a small pot was added to allow the gain to be varied. The preamp had a single integrated circuit and provided a gain of approximately 250 .

\subsection{THE INTERROGATOR}

This unit is the computer or manually controlled device which sends a current pulse down any of the chosen MS lines to initiate the delayed readout. It consists of a logical element which decodes the computer word and an SCR pulser which supplies the current pulse to the MS line.

Each wand had its own SCR pulser; any one of the 56 could be interrogated under computer or manual control. The logical element decoded the 6-bit computer word and used a computer generated flag to fire the chosen SCR pulsers. For test purposes the appropriate pulses could also be selected manually by a switch register.

Studies were made of the optimum size and shape for the interrogating pulse. The shape and amplitude

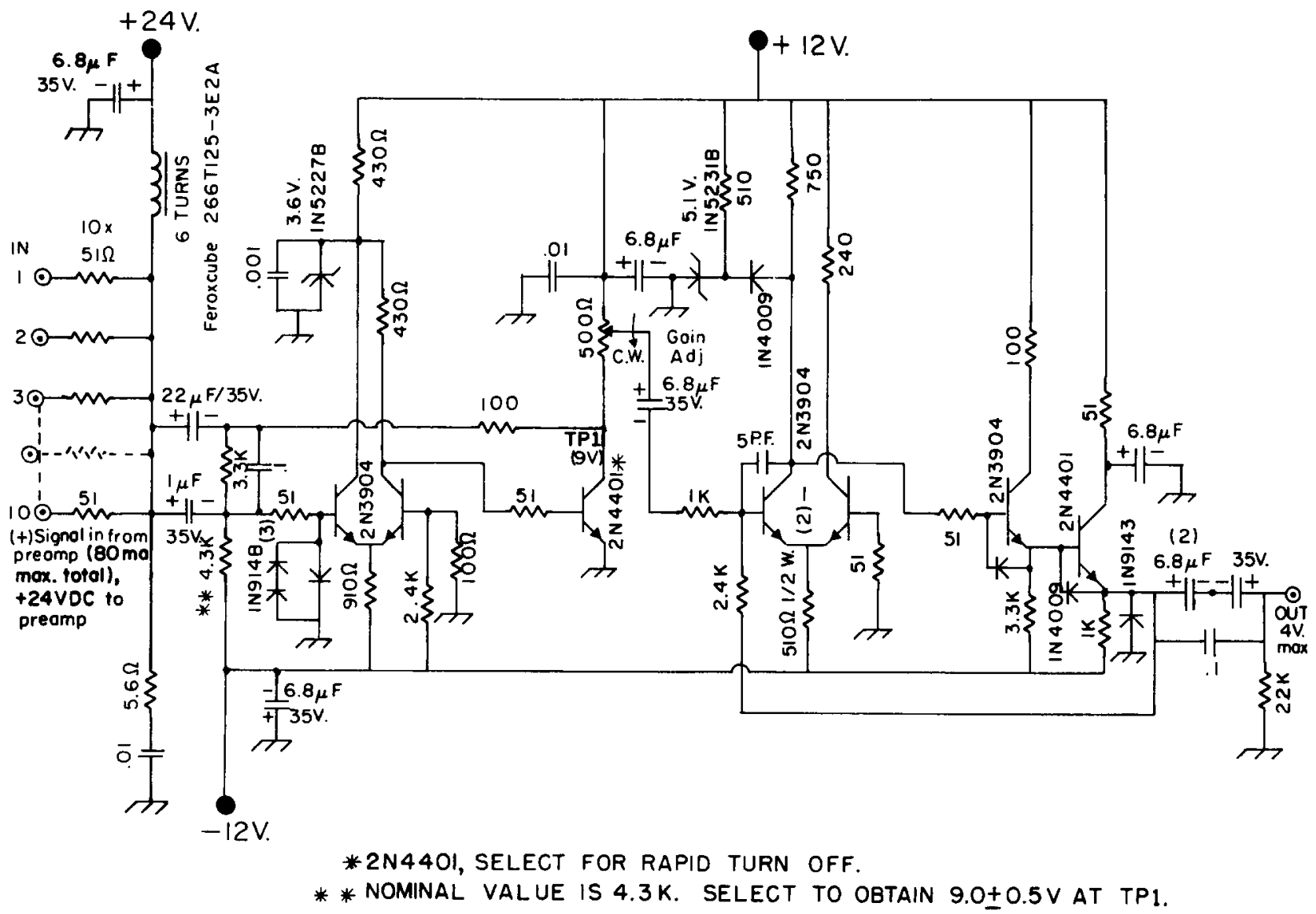

Fig. 5. The 10-input "or" circuit used for summing the preamp outputs. The circuits were mounted in standard single-width NIM modules. The $+24 \mathrm{~V}$ dc power to the preamps was supplied from the "or" circuit. 
of the output pulse height depends to some extent on that of the interrogating pulse. We found that good results with essentially complete erasure could be obtained by discharging a $0.1 \mu \mathrm{F}$ capacitor charged to about $300 \mathrm{~V}$ into the ribbon by means of a simple SCR circuit. The circuit used is shown in fig. 4. A $1 \mathrm{kHz}$ square wave was used to provide $300 \mathrm{~V}$ power to the SCR circuits, insuring SCR turnoff. The interrogators were mounted close to the chambers to minimize cable lengths.

\subsection{OTHER ELECTRONICS}

The outputs of the 56 wands were divided into two banks with all the horizontal wands in one group and the vertical in the other. The outputs of each group were summed by means of the " 10 input or" circuit shown in fig. 5. Three of these were used for the 28 horizontal wands and three for the vertical. They were designed so that they could be cascaded with a fourth circuit used to sum the outputs of the first three. The "ors" also provided the $24 \mathrm{~V}$ dc to the wand preamps through the signal cable. The gain of the circuits could be adjusted between about 0.5 and 4 . The "ors" were mounted close to the chambers so that only two long BNC cables were needed to handle all the wand outputs.

The summed outputs from each group were fed into a discriminator followed by six scalers so that the system had a six spark capability. The discriminator and scalers were standard L.R.L. units ${ }^{4}$ ). A separate discriminator and scaler bank was used for the horizontal and vertical wands, and the logic was

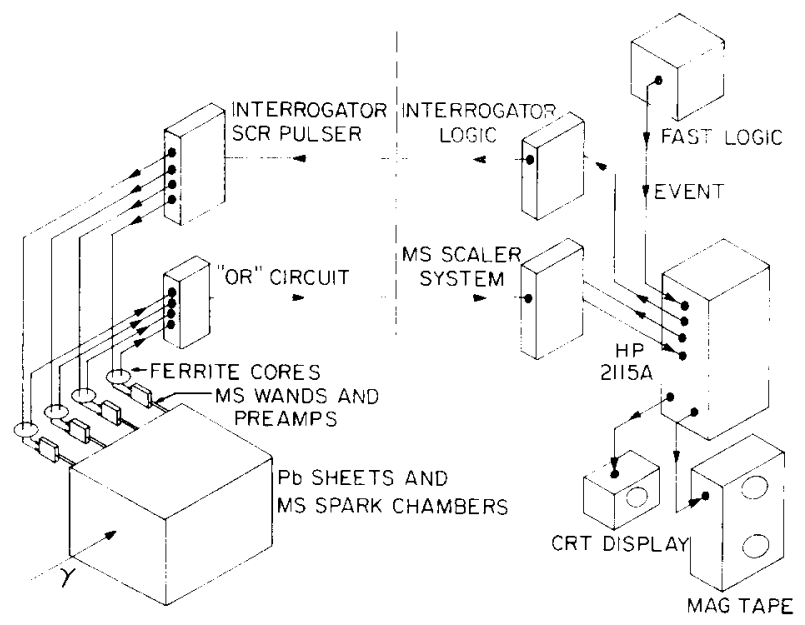

Fig. 6. Block diagram of the readout system. Only a few of the horizontal wands are shown for clarity. arranged so that one horizontal and one vertical wand were interrogated simultaneously.

\subsection{THE READOUT SEQUENCE}

A block diagram of the system is shown in fig. 6 . The sequence of operations was as follows: The chambers were fired. After a short delay to allow the noise to die out, the computer (a Hewlett-Packard $2115 \mathrm{~A}$ ) interrogated the first pair of horizontal and vertical wands. The computer also generated a gate which kept the scalers in reset until just before the pulse from the first fiducial arrived. This eliminated pulses from the current tap and noise generated by the interrogating pulse. After data from the scalers was transferred into core the computer interrogated the next pair of wands. This sequence continued until all 28 pairs of wands were read, after which the system was ready for another event.

The total readout time in our experiment therefore was the sum of the time required to read out each of the 28 horizontal wands, the longer of the two kinds. The total readout time was about $5 \mathrm{msec}$. This, of course, is much longer than the time that would be required to read out a prompt system, but in this experiment the longer readout time was not a consideration.

Thus the computer did all the "bookkeeping" and controlled the entire readout process. The computer also acted as a buffer to the magnetic tape and made displays which were used to monitor the chamber performance.

\section{Performance of the system}

After initial debugging and tuneup the system performed quite well. Some noteworthy problems which were encountered are briefly discussed here. Considerable care with ground currents was needed to keep the SCR's in the interrogators from firing Icm

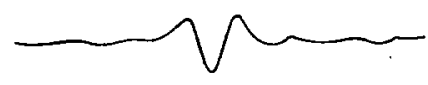

Static

$.005 \mathrm{~V} / \mathrm{cm}$ verticol,

$2 \mu \mathrm{sec} / \mathrm{cm}$ horizontal

Dynomic

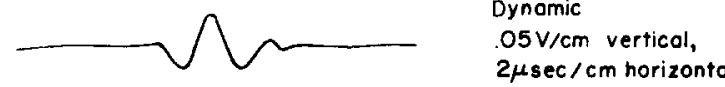

Fig. 7. Comparison of static and dynamic pulses from the same ribbon $(0.10 \times 0.38 \mathrm{~mm})$ measured directly at the pickup coil. The static pulses are typically about one-tenth the dynamic and have opposite polarity. The width of the two are comparable, but considerably narrower dynamic pulses can be obtained with unannealed ribbon of smaller cross section. 


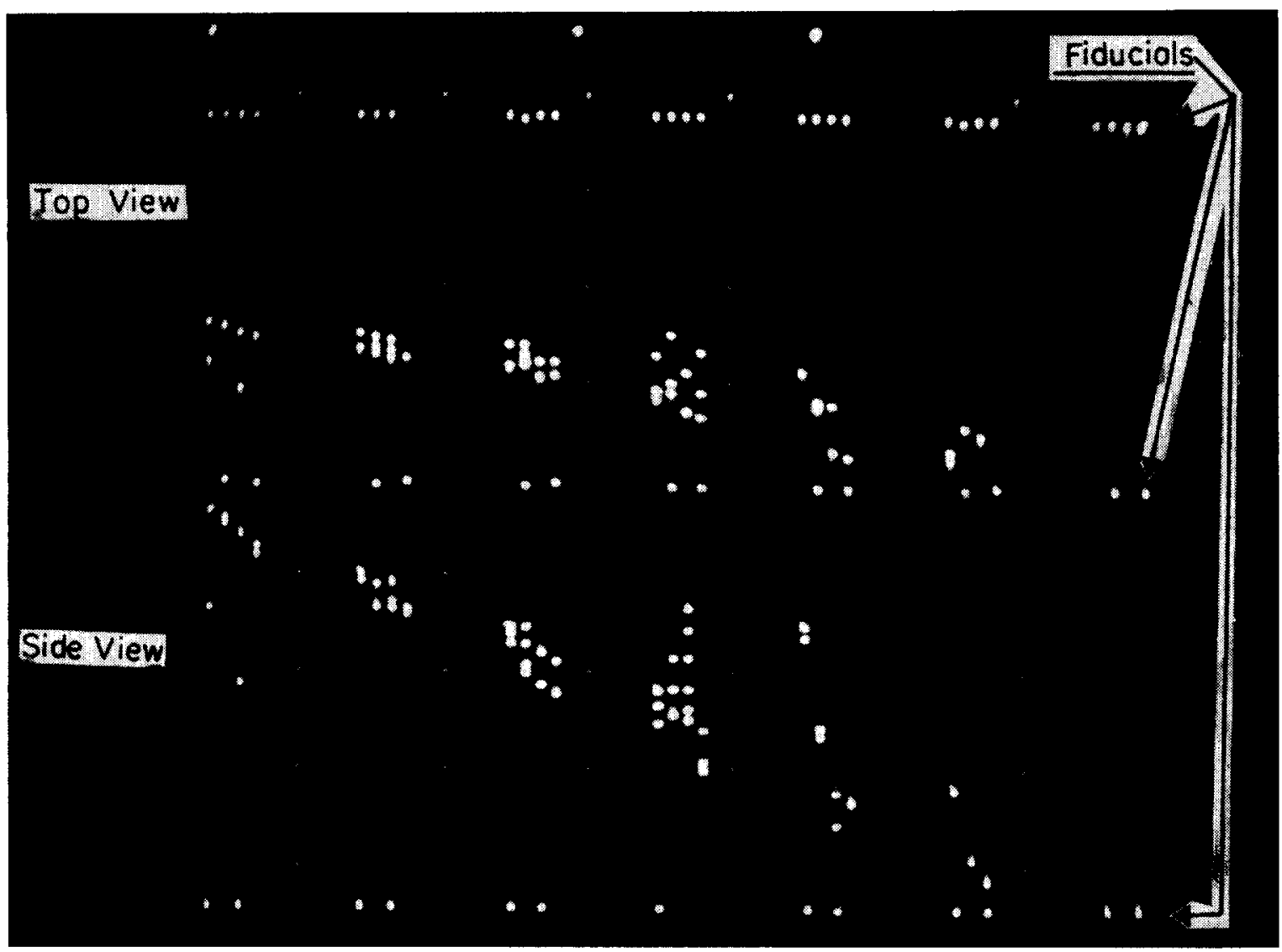

Fig. 8. A cosmic ray shower produced in the chamber array and reconstructed on a computer display. The computer display was intended only for monitoring and was of rather poor quality. The shower originates near the left-hand edge of the array. The dotted vertical lines indicate which of the eight triggering counters in the array fired.

when all the chambers fired. To prevent this, all the BNC interrogator cables had to be routed through a grounded patch panel. It was also found that the ribbons on the H.V. planes of the chambers were being partially erased by current set up when the chambers fired because of the capacitative coupling between the wands and the H.V. plane. This was effectively eliminated by wrapping both BNC cables to the wand several times around a ferrite toroid and taking care that the wands were not otherwise grounded.

As mentioned previously, we do not yet have any direct measurement of the resolution of the static system, but there are indications that it is likely to be somewhat poorer than for the prompt. Fig. 7 compares static and prompt pulses from the same ribbon $(0.10 \times 0.38 \mathrm{~mm})$ measured directly at the pickup coil output. Note that the prompt pulse is approximately 10 times the static pulse in amplitude though both are similar in shape. The width of the central lobe at the baseline is $\approx 1.4 \mu \mathrm{sec}$ for both. With $0.15 \mathrm{~mm}$ unannealed ribbon we have consistently obtained pulse widths $<0.5 \mu \mathrm{sec}$ for the central lobe (see also ref. 4). Pulse shapes also seem to be more consistent with the dynamic readout. The spatial resolution required in the $\mathrm{n}+\mathrm{p} \rightarrow \gamma+\mathrm{d}$ experiment was modest $(\approx 2 \mathrm{~mm})$ and that obtained with the static system was certainly sufficient.

Fig. 8 shows a cosmic ray shower produced in the chambers and reconstructed on a computer-generated display. The upper part of the display shows the "top" view and the lower the "side" view. The second fiducials are also plotted in the display. These are plotted along the top and bottom edges and between the two views. The dashed vertical lines indicate which of triggering counters fired.

It can be seen from fig. 8 that the multitrack efficiency is adequate for good vertex location, the main consideration in the $\mathrm{n}+\mathrm{p} \rightarrow \gamma+\mathrm{d}$ experiment. The absence of spurious sparks in the four gaps on the 
right illustrates that even though the chambers were driven quite hard and no "crowbars" were used, spurious breakdowns in the absence of a track were rare. On the other hand, gap-by-gap comparison of the top and side views shows several discrepancies in spark counts between the two views. This indicates that multiple sparks were not registered with high efficiency. It should be possible to improve this aspect of the chamber performance with further development.

\section{Conclusions}

The static readout system described has been used for digitizing spark coordinates from a gamma shower detector. The system performed quite satisfactorily in an experiment in which $\approx 10^{7}$ events were collected. With the delayed readout we were able to design a system capable of digitizing $6 \times 56$ spark locations per event using 12 scalers instead of the 336 that would have been necessary with a conventional readout system.

Disadvantages of the system include a somewhat greater sensitivity to magnetic fields, longer readout time, and probably poorer spatial resolution than the usual systems with prompt readout. Construction of magnetostrictive lines with adequate signal-to-noise ratio was also considerably more difficult.

We would like to thank O. Haas and R. French for their skillful work in building and testing the chambers. J. Walker and T. Baker were instrumental in building and testing the wands. J. Pluta was responsible for most of the electronic circuits.

\section{References}

1) T. V. Bespalova, I. A. Golutvin, Yu. V. Zanevsky, Yu. T. Kiryushin, V. D. Kondrashov, V. D. Peshekhonov, V. D. Ryabtsov, E. A. Silayev and D. A. Smolin, Intern. Symp. Nuclear electronics, vol. III (La Documentation Française, Versailles, Paris, 1968).

2) F. C. Monds and A. M. Rosie, Proc. IEE 113, no. 12 (Dec. 1966) 1943.

3) We have recently discovered that heat treatment of the receiving end of the ribbon (near the pickup coil) only is sufficient to realize most of the gain in pulse height. Annealing of the remainder of the ribbon is not necessary. It is therefore possible to achieve a large increase in pulse height with negligible effect on the attenuation.

4) F. A. Kirsten, K. L. Lee and J. Conragan, Lawrence Radiation Laboratory Report UCRL-16539 (March 1966) unpublished. 